Mathematical Modelling and Analysis

Volume 19 Number 4, September 2014, 443-449

http://dx.doi.org/10.3846/13926292.2014.936981

(c) Vilnius Gediminas Technical University, 2014
Publisher: Taylor\&Francis and VGTU

http://www.tandfonline.com/TMMA

Print ISSN: 1392-6292

Online ISSN: 1648-3510

\title{
Mathematical Model of Electrodiffusion, Dissociation and Recombination in the Diffusive Layer*
}

\author{
Andrey V. Shobukhov ${ }^{a}$, Nadejda P. Savenkova ${ }^{a}$, \\ Runar N. Kuzmin ${ }^{b}$ and Dmitry S. Maximov ${ }^{c}$ \\ ${ }^{a}$ Faculty of Computational Mathematics and Cybernetics Lomonosov Moscow \\ State University \\ 2-nd Educat. Building, Leninskiye Gory, 119991 Moscow, Russian Federation \\ ${ }^{b}$ Faculty of Physics, Lomonosov Moscow State University \\ Leninskiye Gory, 119991 Moscow, Russian Federation \\ ${ }^{c}$ Moscow Radiotechnical Institute, Russian Academy of Science \\ 132, Varshavskoe shosse, 117519 Moscow, Russian Federation \\ E-mail: shobukhov@cs.msu.su
}

Received August 8, 2013; revised June 11, 2014; published online September 1, 2014

\begin{abstract}
We study a mathematical model of electrochemical processes in the thin diffusive layer of polymer electrolyte between the electrode and the bulk. We consider diffusion, migration, dissociation and recombination of positive and negative ions in the electric field that take place in this region under various boundary conditions. We demonstrate that the unique stable steady state of the electrochemical system is determined by the ratio of dissociation to recombination. This state attracts timedependent solutions of the model, but their convergence strongly depends on the electroneutrality of the bulk.
\end{abstract}

Keywords: ion motion, dissociation, recombination, polymer electrolyte, modeling.

AMS Subject Classification: $81 \mathrm{~T} 80 ; 78 \mathrm{~A} 57$.

\section{Introduction}

The influence of the dissociative and recombinative processes of ions on the migration current has been studied by many authors for various cations, anions and neutral substances $[3,7,10]$. Modeling the formation of concentration gradients in electrolyte adjacent to different interfaces [8] and the response of an electrochemical cell to the applied ac and dc voltage [6] reveals complicated

\footnotetext{
* The authors are very grateful to Dr. Isaac Rubinstein from the Department of Mathematics, Ben-Gurion University of the Negev, for many consultations and fruitful discussions, which have improved this paper in many aspects.
} 
non-equilibrium behavior of ions including the local violation of electroneutrality and the appearance of concentration oscillations in the diffusive layer. In this paper we investigate the waves of ion concentrations that arise under certain conditions in the polymer electrolyte.

Polymer electrolytes make an important class of solid ionic conductors. They are formed as a result of dissolving the alkali metal salts (such as $\mathrm{LiClO}_{4}$ ) in polymers (such as $P E O$, the polyethylene oxide). These electrolytes exhibit strong interactions between the polymer hosts and cations. Simultaneously the cation-anion interactions prove to be also very strong. It leads to significant ion association $[1,2,4,5]$. Experimental data from $[4,5]$ suggest that the neutral ion pairs greatly outnumber single ions. Nevertheless there is strong evidence obtained by various spectroscopic methods $[1,2]$ that simultaneously in $\mathrm{PEO}$ there exist separate cations $\left(\mathrm{Li}^{+}\right)$, anions $\left(\mathrm{ClO}_{4}^{-}\right)$and recombined pairs $\left(\mathrm{LiClO}_{4}\right)$. As a result, in spite of several attempts to explain the ion transport in polymer electrolytes $[4,5]$, its mechanism remains unclear.

To clarify this important problem we propose a simple mathematical model describing transport and reactions of charged species in polymer electrolyte. This model includes diffusion, migration, dissociation and recombination of ions in the diffusive layers close to the electrodes' surfaces. The previous models $[4,5]$ deal mainly with the steady state solutions and do not consider the establishing stage, which may be very long.

\section{The Model}

Following $[4,5]$, in our model we invoke the following basic assumptions:

Assumption 1. The electrodes and the electrolyte between them are planar symmetric.

Assumption 2. The electrolyte consists of cations $\mathrm{Li}^{+}$, anions $\mathrm{ClO}_{4}^{-}$, ion pairs $\mathrm{LiClO}_{4}$ and the polymer, the concentration of the latter remains constant.

Assumption 3 . The total salt concentration $C_{\max }$ is a constant parameter, while the concentration of cations $P=P(t, x)$ and the concentration of anions $N=N(t, x)$ depend both on time $\mathrm{t}$ and on distance from the electrode surface $x$.

Assumption 4. The cations, anions and ion pairs are not assumed to be in equilibrium. They dissociate and recombine according to the scheme:

$$
\begin{aligned}
& K_{D}: \mathrm{LiClO}_{4} \rightarrow \mathrm{Li}^{+}+\mathrm{ClO}_{4}^{-}, \\
& K_{R}: \mathrm{LiClO}_{4} \leftarrow \mathrm{Li}^{+}+\mathrm{ClO}_{4}^{-},
\end{aligned}
$$

where $K_{D}$ and $K_{R}$ denote the dissociation and recombination rates. The molar production rates for $\mathrm{Li}^{+}$and $\mathrm{ClO}_{4}^{-}$equal $K_{D}\left(C_{\max }-\mathrm{N}-\mathrm{P}\right)-K_{R} P N$ while the same rate for $\mathrm{LiClO}_{4}$ equals $-K_{D}\left(C_{\max }-N-P\right)+K_{R} P N$. 
The component mass balance is based on Assumptions 1-4. Together with the Poisson equation for the electric potential $\Phi=\Phi(t, x)$, it gives us the system:

$$
\begin{aligned}
\frac{\partial P}{\partial t}= & D_{P} \frac{\partial}{\partial x}\left(\frac{\partial P}{\partial x}+\frac{F}{R T} P \frac{\partial \Phi}{\partial x}\right) \\
& +K_{D}\left(C_{\max }-N-P\right)-K_{R} P N ; \\
\frac{\partial N}{\partial t}= & D_{N} \frac{\partial}{\partial x}\left(\frac{\partial N}{\partial x}-\frac{F}{R T} N \frac{\partial \Phi}{\partial x}\right) \\
& +K_{D}\left(C_{\max }-N-P\right)-K_{R} P N ; \\
\frac{\partial^{2} \Phi}{\partial x^{2}}= & \frac{F}{\varepsilon_{0} \varepsilon}(N-P) ; \quad 0<x<L .
\end{aligned}
$$

The meanings and values of all coefficients may be found below in Table 1 . This system is supplemented with the boundary conditions:

$$
\begin{gathered}
\frac{\partial P}{\partial x}=0 ; \quad \frac{\partial N}{\partial x}=0 ; \quad \Phi=-\Phi^{*}=\text { const } \quad \text { at } x=0 ; \\
P=P^{*}=\text { const }, \quad N=N^{*}=\text { const }, \quad \Phi=0 \quad \text { at } x=L .
\end{gathered}
$$

These conditions mean: 1) diffusive ion flows at the left boundary (electrode) are absent, 2) cation and anion concentrations at the right boundary (bulk) are constant, 3) potential difference between the electrode and the bulk is fixed. Let us represent the diffusion coefficients as $D_{P}=D \cdot \delta_{P}, D_{N}=D \cdot \delta_{N}$, where $D$ is the scaling factor. Then we may normalize the system (2.1)-(2.5) as follows:

$$
\begin{aligned}
& P=C_{\max } \cdot p, \quad N=C_{\max } \cdot n, \quad \Phi=\frac{R T}{F} \varphi, \quad x=L \cdot \xi, \quad t=\frac{L^{2}}{D} \tau, \\
& K_{R}=\frac{D}{L^{2} \cdot C_{\max }} \cdot k_{R}, \quad K_{D}=\frac{D}{L^{2}} \cdot k_{D}, \quad \mu=\frac{\varepsilon_{0} \varepsilon R T}{C_{\max } \cdot L^{2} \cdot F^{2}} .
\end{aligned}
$$

Further on we shall study our model in the dimensionless variables $(\tau, \xi)$ :

$$
\begin{gathered}
\dot{p}-\delta_{P}\left(p^{\prime}+p \cdot \varphi^{\prime}\right)^{\prime}=k_{D}(1-p-n)-k_{R} p n ; \\
\dot{n}-\delta_{N}\left(n^{\prime}-n \varphi^{\prime}\right)^{\prime}=k_{D}(1-p-n)-k_{R} p n ; \\
\mu \varphi^{\prime \prime}=n-p ; \\
p^{\prime}=0 ; \quad n^{\prime}=0 ; \quad \varphi=-\varphi^{*} \quad \text { for } \xi=0 . \\
p=p^{*} ; \quad n=n^{*} ; \quad \varphi=0 \quad \text { for } \xi=1 .
\end{gathered}
$$

Here $\cdot$ stands for $\partial / \partial \tau$ and ${ }^{\prime}-$ for $\partial / \partial \xi$. It should be mentioned that functions in the right hand sides of (2.1') and (2.2') are exactly the same:

$$
f(p, n)=k_{D}(1-p-n)-k_{R} p n .
$$

\section{Exact Steady State Solution}

Suppose that $p=n=c$, where $c \equiv$ const is the root of the equation $f(c, c)=0$. Together with $\varphi(\xi)=\varphi^{*} \cdot(\xi-1)$, this $p=n=c$ gives us a steady state solution to $\left(2.1^{\prime}\right)-\left(2.5^{\prime}\right)$. There are two roots of $f(c, c)=0$ : 


$$
c_{ \pm}=\frac{k_{D}}{k_{R}}\left( \pm \sqrt{1+\frac{k_{R}}{k_{D}}}-1\right) \text {. }
$$

Being a value of ion concentration, the root should satisfy: $0 \leq c \leq 1$. It is easy to see that $c_{-}$is negative and thus is not suitable, but $c_{+}$always satisfies given condition, since the rates $k_{R}$ and $k_{D}$ are positive due to their physical meaning. And thus (2.1')-(2.5') always has a steady state solution:

$$
p_{0}(\tau, \xi)=n_{0}(\tau, \xi)=c^{*} \equiv \frac{k_{D}}{k_{R}}\left(\sqrt{1+\frac{k_{R}}{k_{D}}}-1\right), \quad \varphi_{0}(\tau, \xi)=\varphi^{*} \cdot(\xi-1) .
$$

To study the stability of this solution we put:

$$
\begin{gathered}
p(\tau, \xi)=c^{*}+\tilde{p}(\tau, \xi) ; \quad n(\tau, \xi)=c^{*}+\tilde{n}(\tau, \xi) ; \quad \varphi(\tau, \xi)=\varphi^{*} \cdot(\xi-1)+\tilde{\varphi}(\tau, \xi) \\
\tilde{p}, \tilde{n}, \tilde{\varphi} \text { are small perturbations of } p, n, \varphi
\end{gathered}
$$

and linearize $\left(2.1^{\prime}\right)-\left(2.5^{\prime}\right)$ in its vicinity. After substituting $\varphi^{\prime \prime}$ from (2.3') into $\left(2.1^{\prime}\right)-\left(2.2^{\prime}\right)$ and discarding the nonlinear terms, we obtain:

$$
\begin{aligned}
& \dot{\tilde{p}}=\delta_{P}\left(\tilde{p}^{\prime \prime}+\varphi^{*} \tilde{p}^{\prime}+\frac{c^{*}}{\mu}(\tilde{n}-\tilde{p})\right)-k_{D}(\tilde{p}+\tilde{n})-k_{R} c^{*} \cdot(\tilde{p}+\tilde{n}) \\
& \dot{\tilde{n}}=\delta_{N}\left(\tilde{n}^{\prime \prime}-\varphi^{*} \tilde{n}^{\prime}-\frac{c^{*}}{\mu}(\tilde{n}-\tilde{p})\right)-k_{D}(\tilde{p}+\tilde{n})-k_{R} c^{*}(\tilde{p}+\tilde{n}) .
\end{aligned}
$$

For checking the stability of (3.1) we seek the solution to (3.2) in form of:

$$
\left(\begin{array}{l}
\tilde{p}(\tau, \xi) \\
\tilde{n}(\tau, \xi)
\end{array}\right)=\left(\begin{array}{l}
u \\
\nu
\end{array}\right) \exp (\sigma \tau+i k \xi)
$$

and obtain a SLAE with respect to $(u, v)$. The solvability condition for this SLAE is a quadratic equation with respect to $\sigma$; its both roots are real and negative. Therefore $(\tilde{p}, \tilde{n})$ tend to zero when $\tau \rightarrow \infty$, and thus the solution (3.1) is unconditionally stable. It should be the attractor for time-dependent solutions to $\left(2.1^{\prime}\right)-\left(2.5^{\prime}\right)$. We demonstrate this feature numerically in the next section.

\section{Numerical Results and Discussion}

In this section we present the results of numerical solving of $\left(2.1^{\prime}\right)-\left(2.5^{\prime}\right)$. We use the symmetric implicit finite difference scheme which has the 2-nd order of accuracy with respect both to $\tau$ and to $\xi$. The mesh contains $10^{4}$ nodes in $\xi$; the time step $\Delta \tau$ is taken equal $10^{-2} \Delta \xi$; the obtained system of nonlinear equations is solved with Seidel-type iterations. The parameters of the model are presented in Table $1[1,2,4,5]$.

Initially we suppose that the bulk is electroneutral, which means that $p^{*}=n^{*}$ in the boundary condition $\left(2.5^{\prime}\right)$. In this case the time-dependent solution converges to the steady state distribution of $p, n$ and $\varphi$ very quickly. If $p^{*}=n^{*}=c^{*}$ then we immediately obtain the constant solution (3.1). In the 
Table 1. Constants and parameter values of the model.

\begin{tabular}{lll}
\hline Symbol & Physical meaning & Value \\
\hline$L$ & Thickness of the diffusive layer & $1.00 \mathrm{E}-05 \mathrm{~m}$ \\
$C_{\max }$ & Maximal alkali salt concentration & $2.84 \mathrm{E}+03 \mathrm{~mol} \cdot \mathrm{m}^{-3}$ \\
$D$ & Diffusion scaling factor & $1.00 \mathrm{E}-09$ \\
$\delta P$ & Normalized diffusion coefficient of $\mathrm{Li}^{+}$ & $7.00 \mathrm{E}-03 \mathrm{~m}^{2} \cdot \mathrm{s}^{-1}$ \\
$\delta N$ & Normalized diffusion coefficient of $\mathrm{ClO}_{4}^{-}$ & $3.30 \mathrm{E}-02 \mathrm{~m}^{2} \cdot \mathrm{s}^{-1}$ \\
$K_{D}$ & Rate constant for $\mathrm{LiClO}_{4}$ dissociation & $1.03 \mathrm{E}+03 \mathrm{~s}^{-1}$ \\
$K_{R}$ & Rate constant for $\mathrm{LiClO}_{4}$ recombination & $2.71 \mathrm{E} 01 \mathrm{~m}^{3} \cdot \mathrm{mol}^{-1} \cdot \mathrm{s}^{-1}$ \\
$T$ & Absolute temperature & $4.03 \mathrm{E}+02 \mathrm{~K}$ \\
$\varepsilon$ & Relative permittivity & $1.00 \mathrm{E}+00$ \\
$\varepsilon_{0}$ & Absolute permittivity & $8.85 \mathrm{E}-12 \mathrm{~F} \cdot \mathrm{m}^{-1}$ \\
$R$ & Universal gas constant & $8.31 \mathrm{~J} \cdot \mathrm{mol}^{-1} \cdot \mathrm{K}^{-1}$ \\
$F$ & Faraday constant & $9.6487 \mathrm{E}+04 \mathrm{C}^{-1} \mathrm{~mol}^{-1}$ \\
\hline
\end{tabular}

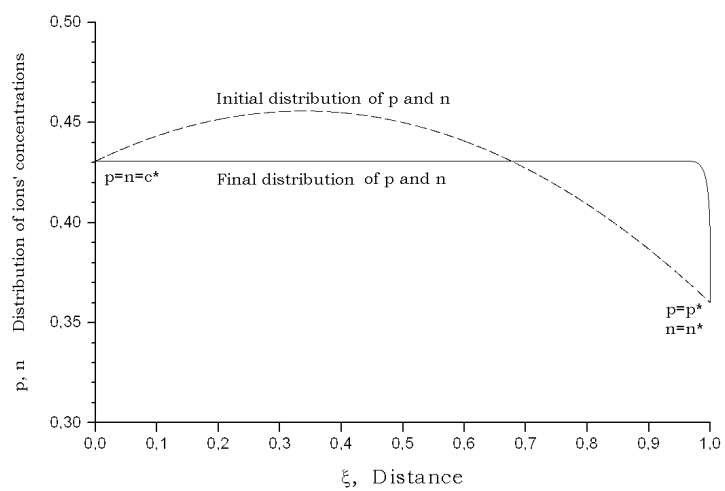

Figure 1. The steady state distribution: $p^{*}=n^{*}$, but $p^{*} \neq c^{*}, n^{*} \neq c^{*}$. The curves $p=p(\xi)$ and $n=n(\xi)$ coincide with each other.

case of $p^{*}=n^{*}$ with $p^{*} \neq c^{*}$ and $n^{*} \neq c^{*}$ the bulk is also electroneutral, but its state differs from the equilibrium of the diffusive layer. As a result, by the moment of $\tau=10^{-4}$ we get the final distribution of $p$ and $n$ with a boundary layer at $\xi=1$ (see Fig. 1).

Let us denote the current density $J$ as the difference between the flows of positive and negative ions through the unit electrode surface at $\xi=0$. Given the boundary condition $\left(2.4^{\prime}\right), J$ may be evaluated as follows:

$$
J=\left.\left(\delta_{P} \cdot\left(p^{\prime}+p \cdot \varphi^{\prime}\right)-\delta_{N} \cdot\left(n^{\prime}-n \varphi^{\prime}\right)\right)\right|_{\xi=0}=\left.\left(\left(\delta_{P} p-\delta-N \cdot n\right) \varphi^{\prime}\right)\right|_{\xi=0} .
$$

During the formation of the final distribution $J$ exhibits a single pulse. After that it remains constant, as it is shown in Fig. 2. This pulse corresponds to the short establishing period for the ions' steady state concentrations.

The process becomes completely different with $p^{*} \neq n^{*}$, when the electroneutrality of the bulk is not supported (it may happen in very long and narrow pores [9], though the authors of [9] do not consider such possibility in their model). Besides the boundary layer at $\xi=1$, we now observe the on- 


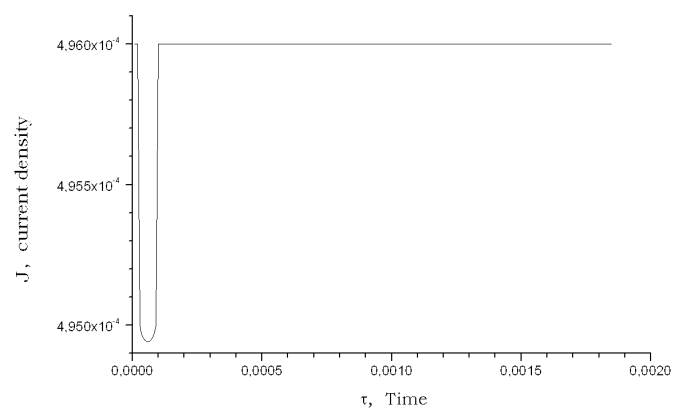

Figure 2. Current density $J=J(\xi)$ at the electrode surface. Electroneutral bulk.

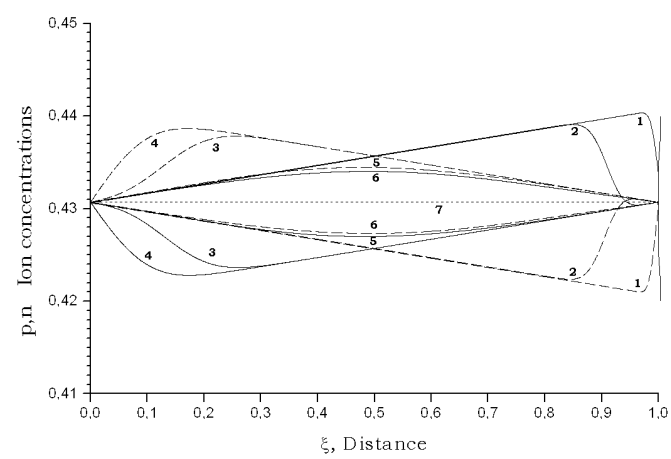

Figure 3. The concentration waves: $p^{*} \neq n^{*}, p^{*} \neq c^{*}$ and $n^{*} \neq c^{*}$. Solid line: $p=p(\tau, \xi)$. Dashed line: $n=n(\tau, \xi) .1: \tau=10,2: \tau=100,3: \tau=900,4: \tau=100$, 5: $\tau=9000,6: \tau=10000,7:$ equilibrium.

set of concentration oscillations. Slowly attenuating waves of $p=p(\tau, \xi)$ and $n=n(\tau, \xi)$ are shown in Fig. 3. Simultaneously the current density oscillations occur, as it is shown in Fig. 4.

Concentration waves in Fig. 3 fade and finally approach the steady state solution which practically coincides with the equilibrium (3.1), except for the vicinity of $\xi=1$. There the steady state solution exhibits the boundary layer, and concentrations reach the prescribed values $\left(p^{*}=0.44, n^{*}=0.42\right)$.

\section{Conclusions}

We have proposed and studied a mathematical model of the ion dissociation, recombination, diffusion and migration in the polymer electrolyte diffusive layer. We have proved that when the electrolyte bulk is electroneutral, then under certain boundary conditions there exists a unique steady state, which is unconditionally stable. Numerically we have demonstrated that when the electroneutrality of the bulk is not supported, then the model exhibits concentration waves that cause the current density oscillations at the electrode surface. These oscillations may be observed in physical experiments. 


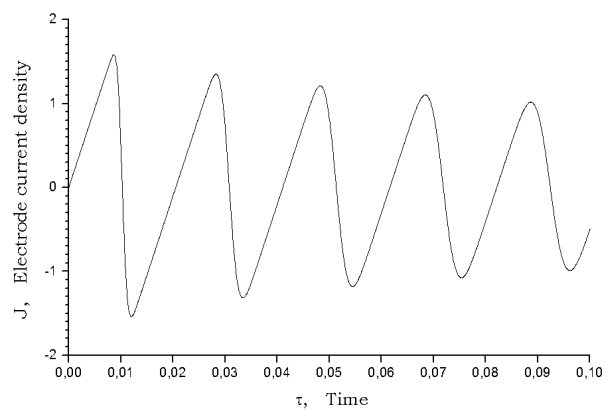

Figure 4. Current density $J=J(\tau)$ at the electrode surface. Non-electroneutral bulk.

\section{References}

[1] M. Furlani, C. Stappen, B.E. Mellander and G.A. Niklasson. Concentration dependence of ionic relaxation in lithium doped polymer electrolytes. J. NonCrystalline Solids, 356:710-714, 2010.

http://dx.doi.org/10.1016/j.jnoncrysol.2009.07.039.

[2] A. Karmakar and A. Ghosh. Dielectric permittivity and electric modulus of polyethylene oxide $(\mathrm{PEO})-\mathrm{LiClO}_{4}$ composite electrolytes. Cur. Appl. Physics, 12:539-543, 2012. http://dx.doi.org/10.1016/j.cap.2011.08.017.

[3] Yu.I. Kharlats and A.V. Sokirko. Theory of the effect of migration current exaltation taking into account dissociation-recombination reactions. J. Electroanal. Chem., 303:27-44, 1991. http://dx.doi.org/10.1016/0022-0728(91)85113-4.

[4] C. Lin, R.E. White and H.J. Ploehn. Modeling the effects of ion association on direct-current polarization of solid polymer electrolytes. J. Electrochem. Soc., 147(3):936-944, 2000. http://dx.doi.org/10.1149/1.1393295.

[5] C. Lin, R.E. White and H.J. Ploehn. Modeling the effects of ion association on alternating current impedance of solid polymer electrolytes. J. Electrochem. Soc., 149(7):E242-E251, 2002. http://dx.doi.org/10.1149/1.1480018.

[6] L.H. Oesen, M.Z. Bazant and H. Bruus. Strongly nonlinear dynamics of electrolytes in large ac voltages. Phys. Rev. E, 82:011501(29), 2010.

[7] I. Rubinstein. Effects of deviation from local electroneutrality upon electrodiffusional ionic transport across a cation-selective membrane. Reactive Polymers, 2:117-131, 1984.

[8] I. Rubinstein and B. Zaltzman. Electro-osmotically induced convection at a permselective membrane. Phys. Rev. E, 62(2):2238-2251, 2000. http://dx.doi.org/10.1103/PhysRevE.62.2238.

[9] M. Verbrugge and P. Liu. Microstructural analysis and mathematical modeling of electric double-layer supercapacitors. J. Electrochem. Soc., 152(5):D79-D87, 2005. http://dx.doi.org/10.1149/1.1878052.

[10] V.I. Zabolotskii, V.V. Nikonenko, N.M. Korzhenko, R.R. Seidov and M.K. Urtenov. Mass transfer of salt ions in an electrochemical system with violated electroneutrality in the diffusion layer: The effect of a heterolytic dissociation of water. Russian J. Electrochem., 38(8):810-818, 2002.

http://dx.doi.org/10.1023/A:1016849309018. 